\title{
Drugs acting on the heart: heart failure and coronary insufficiency
}

- Takashi Kudo, MD is Honorary Visiting Fellow in the Department of Cardiovascular Sciences, University of Leicester, Leicester Royal Infirmary, Leicester, UK. Conflicts of interest: none declared.

- Jonathan P Thompson, BSc (Hons) MD FRCA FFICM is Consultant in Anaesthesia and Critical Care, University Hospitals of Leicester NHS Trust and \& Honorary Senior Lecturer, University of Leicester, Leicester Royal Infirmary, Leicester, UK. Conflicts of interest: none declared.

\section{Learning objectives}

After reading this article, you should be able to:

- define heart failure

- list management strategies for acute heart failure

- list drugs used in chronic heart failure

- describe the role of anti-platelet drugs and statins in coronary artery disease

\begin{abstract}
:
Heart failure (HF) and coronary insufficiency are common amongst surgical and critical care patients. Both are chronic conditions interrupted by acute episodes. HF activates neurohormonal mechanisms that worsen renal and cardiac function. Acute heart failure (AHF) commonly presents with dyspnoea as a consequence of systolic and / or diastolic dysfunction. Goals of treatment are symptom relief, to maintain tissue perfusion and optimise cardiac function. Diuretics and vasodilators are used early; inotropes are reserved for when other treatment has failed.
\end{abstract}

Chronic heart failure (CHF) is treated using changes in lifestyle and drugs to manage symptoms. ACE inhibitors and beta-blockers are effective in systolic heart failure and are associated with improved mortality. HF with preserved ejection fraction (HFPEF) is less responsive to drug therapy, though outcomes are better than for systolic HF.

Coronary insufficiency occurs when myocardial oxygen delivery is inadequate, leading to symptoms of ischaemic heart disease (IHD). Treatment goals are maintaining coronary blood flow and reducing myocardial oxygen demand. Beta-blockers and anti-platelet drugs improve outcomes; modern antiplatelets are more effective but associated with risks of haemorrhage. Statins are effective for primary and secondary prevention of myocardial infarction; they have additional anti-inflammatory properties.

Keywords: heart failure, coronary artery disease, heart drugs, heart failure with preserved ejection fraction. 


\section{Heart Failure}

Heart failure (HF) is the inability of the heart to maintain sufficient blood flow to the tissues to meet physiological requirements. It is a clinical syndrome that combines symptoms of heart failure (e.g. dyspnoea), signs of fluid retention (e.g. ankle swelling) and objective evidence of structural or functional abnormalities of the heart at rest (e.g. abnormal echocardiogram, third heart sound). ${ }^{1}$ Heart failure can be further classified according to the clinical presentation into new onset, transient (symptoms occur over a limited time period) and chronic (including decompensated) heart failure. The cause and presentation of heart failure should be sought as they govern treatment.

\section{Acute heart failure (AHF)}

Acute heart failure (whether new onset or decompensated) is characterised by the development of a rapid onset or change in the signs and symptoms of $\mathrm{HF}$, especially dyspnoea. It is caused by left ventricular (LV) systolic dysfunction, diastolic dysfunction, or more commonly a combination of both. Systolic dysfunction is a consequence of impaired myocardial contractility, leading to reduced left ventricular ejection fraction (LVEF) and consequently reduced cardiac output. Diastolic dysfunction is caused by an increase in ventricular stiffness and impaired relaxation, resulting in impaired ventricular filling during diastole; LVEF may be normal. Decreased cardiac output leads to decreased blood flow and tissue perfusion which activates neurohormonal mechanisms leading to renal and myocardial dysfunction (Figure 1).

The goals of treatment in AHF are to maintain adequate peripheral perfusion, improve myocardial contractility and reduce fluid overload. Supplemental oxygen should be administered to all hypoxic patients; diuretics should be given early if there are signs of pulmonary congestion and vasodilators if there is dyspnoea at rest. However, it is important to identify the aetiology of the cardiac dysfunction and treat any precipitating factors. For example, in patients with AHF caused by diastolic dysfunction and with normal or high arterial pressure, vasodilators and continuous positive airway pressure (CPAP) or assisted non-invasive ventilation (NIV) should be used early in the course of treatment. Diuretics are indicated only if there is evidence of fluid overload. Conversely, in patients with cardiogenic shock, positive inotropic drugs should be given with intravenous fluids guided to maintain cardiac output; however in patients with isolated right ventricular failure, diuretics are the mainstay of therapy.

There has been much research aimed at developing new treatments for AHF in the last few decades and guidelines have been produced. Several newer drugs have been shown to improve symptoms of AHF, but without convincing effects on long-term outcome. In addition, it is worth noting that there are remarkably few good randomised data to support the use of traditional therapies. 


\begin{tabular}{|c|c|c|c|}
\hline Therapeutic Goal & Drug & Mode of Action & Side-effects \\
\hline Systemic congestion & $\begin{array}{l}\text { Diuretics e.g. } \\
\text { furosemide, } \\
\text { metolazone }\end{array}$ & $\begin{array}{l}\text { Diuresis and variable } \\
\text { natriuresis }\end{array}$ & $\begin{array}{l}\text { Hypotension, } \\
\text { neurohumoral } \\
\text { activation, electrolyte } \\
\text { abnormalities, renal } \\
\text { dysfunction }\end{array}$ \\
\hline Vasodilation & Nitrates e.g. GTN, SNP & $\begin{array}{l}\text { Venodilation, } \\
\text { vasodilation }\end{array}$ & $\begin{array}{l}\text { Profound hypotension } \\
\text { with vasodilators, } \\
\downarrow \text { coronary perfusion } \\
\text { (SNP), headache, } \\
\text { metabolite toxicity }\end{array}$ \\
\hline Cardiac function & $\begin{array}{l}\text { Inotropes: } \beta \text {-agonists, } \\
\text { PDE-3 inhibitors, } \\
\text { levosimendan }\end{array}$ & $\begin{array}{l}\text { Increased force of } \\
\text { myocardial contraction } \\
+ \text { peripheral } \\
\text { vasodilation } \\
\text { (inodilation) }\end{array}$ & $\begin{array}{l}\text { Increased myocardial } \\
\text { oxygen demand, } \\
\text { hypotension, } \\
\text { arrythmias }\end{array}$ \\
\hline Cardiac stability & $\begin{array}{l}\text { Beta-blockers: } \\
\text { emololol, metoprolol } \\
\text { digoxin }\end{array}$ & $\begin{array}{l}\text { Reduction in heart rate } \\
\text { \& sympathetic activity; } \\
\text { anti-arrhythmic }\end{array}$ & $\begin{array}{l}\text { Bradycardia, } \\
\text { hypotension }\end{array}$ \\
\hline
\end{tabular}

Table: Pharmacological strategies for the emergency management of AHF (adapted from Khan et al, 2008)

Key: AHF, acute heart failure; GTN, glyceryl trinitrate; PDE, phosphodiesterase; SNP, sodium nitroprusside.

\section{Drugs used in AHF}

Opioids

Morphine $(2.5-5 \mathrm{mg})$ is recommended in Europe ${ }^{1}$ as an early treatment where there is chest pain, dyspnoea or restlessness at presentation. Opioids reduce preload through venodilation, reduce afterload and lower heart rate. Dyspnoea and agitation are also improved. Opioids should however be used with caution because of their side effects of central nervous and respiratory depression.

\section{Diuretics}

Diuretics are the mainstay of treatment for acute and chronic heart failure, effectively relieving systemic or pulmonary venous congestion. Furosemide (a loop diuretic) blocks the $\mathrm{Na}^{+} / \mathrm{K}^{+} / 2 \mathrm{Cl}^{-}$cotransporter at the loop of Henle, inhibiting sodium reabsorption and resulting in diuresis. It also acts as a venodilator to produce early relief from dyspnoea. It should be administered promptly $(20-40$ $\mathrm{mg}$ i.v.) and may also be administered as an infusion, with the aim of a weight reduction of approximately $1 \mathrm{~kg}$ per day. The maximum dose should not exceed $100 \mathrm{mg}$ in the first 6 hours as adverse effects (e.g. otoxocity, hyponatraemia, hypokalaemia and dehydration) predominate without additional benefit.

The combination of a loop diuretic and thiazides or related diuretics (e.g. metolazone) is more effective than high doses of loop diuretics alone, and also reduces adverse effects.. Intravenous infusions allow higher doses to be administered with a lower incidence of ototoxicity.

\section{Vasodilators}


Intravenous nitrates are recommended in patients with pulmonary oedema and a systolic pressure $>110 \mathrm{mmHg}$ and in the absence of significant mitral or aortic stenosis, though it is recognised that therapy with nitrates does not necessarily improve outcomes. Glyceryl trinitrate (GTN) is used most commonly $(10-1000 \mu \mathrm{g} / \mathrm{min}$, starting at lower doses). GTN indirectly produces nitric oxide (NO) which causes smooth muscle relaxation, predominately of venous capacitance vessels. This reduces left ventricular filling pressure, making GTN effective where there is diastolic dysfunction.

Predominant systolic dysfunction is effectively treated with sodium nitroprusside (SNP; starting dose $0.3 \mu \mathrm{g} / \mathrm{kg} / \mathrm{min}$ ). SNP is a direct donor of NO and potent vasodilator.

Vasodilators may cause precipitous drops in blood pressure and reduce coronary blood flow. Acute tolerance (tachyphylaxis) also occurs to GTN within $24-48$ hours, probably caused by substrate depletion, enhanced vasoconstrictor activity and upregulation of intracellular phosphodiesterases. SNP is metabolised to toxic products including cyanide and sodium thiocyanate. These toxins disrupt the electron transport chain through inhibition of cytochrome c oxidase, preventing aerobic ATP production. This may result in tinnitus, nausea, abdominal pain, mental changes and ultimately coma and death. The dose and duration of treatment with SNP should be limited $(10-200 \mu \mathrm{g} / \mathrm{min}$ for a maximum of 3 days), particularly in the presence of impaired renal function.

In $\mathrm{HF}$ the secretion of natriuretic peptides A and B (ANP and BNP) by the atrial and ventricular myocardial cells is increased. These peptides act on the endothelium and vascular smooth muscle to cause vasodilation and decrease cardiac filling pressures. Additionally, they inhibit renin and aldosterone production, causing mild natriuresis, and increase both cardiac output and coronary blood flow. Nesiritide is a human recombinant B-type natriuretic peptide that mimics endogenous natriuretic peptide $B(B N P)$ improving symptoms. Its role in the management of AHF is debatable - a large randomised controlled trial (RCT) demonstrated a significant increase in hypotension with no improvement in survival or rates of hospitalisation. ${ }^{2}$

\section{Positive Inotropes}

Positive inotropes are reserved for patients who have either failed or displayed a limited response to vasodilator and diuretic therapy; are hypotensive (systolic pressure $<90 \mathrm{~mm} \mathrm{Hg}$ ); or have signs of tissue hypoperfusion (cold and clammy skin, metabolic acidosis, renal failure and altered mental activity) caused by low cardiac output. The most commonly used positive inotropes are dobutamine, milrinone, or enoximone; none have been shown to improve outcome in AHF.

$\beta_{1}$-adrenoceptor agonists (e.g. dobutamine) increase intracellular cyclic adenylate monophosphate (cAMP), causing the release of calcium from the sarcoplasmic reticulum, thereby increasing cardiac muscle contractile force. Their effects are both chronotropic and inotropic, though hypotension may occur due to $\beta_{2}$-mediated vasodilation. Dobutamine therapy $(2.5 \mu \mathrm{g} / \mathrm{kg} / \mathrm{min}-10 \mu \mathrm{g} / \mathrm{kg} / \mathrm{min})$ is limited by tachycardia, arrhythmias and myocardial ischaemia.

Inhibition of phosphodiesterase (PDE) prevents the catalysis of cAMP thereby increasing intracellular levels. The isoenzyme PDE-3 is found in myocardial cells as well as in vasculature and platelets and is antagonised by drugs such as milrinone and enoximone. These PDE-3 inhibitors increase cardiac output and reduce filling pressures, and cause less tachycardia, fewer arrhythmias and smaller increases in myocardial oxygen demand compared with dobutamine; pulmonary and systemic vascular resistance are reduced to a similar degree. These drugs are usually administered as infusions in the acute setting. Due to their prolonged half-lives (milrinone $1-2$ hours; enoximone 4 -6 hours), loading doses are necessary ( $50 \mu \mathrm{g} / \mathrm{kg}$ milrinone; $90 \mu \mathrm{g} / \mathrm{kg} / \mathrm{min}$ enoximone). Heart failure of an ischaemic cause is a relative contraindication to their use. 
Levosimendan is a newer inotrope that binds to troponin-C, sensitising myocardial microfilaments to calcium and thereby increasing cardiac output whilst reducing filling pressures. Levosimendan also facilitates adenosine triphosphate (ATP)-dependent potassium channel opening causing vasodilation which reduces preload and afterload. Following data from a large-scale RCT where there was no mortality benefit over dobutamine, there are no current plans for its marketing in the UK.

Vasopressors (e.g. noradrenaline) may be required in AHF to increase arterial pressure where there is persistent end-organ hypoperfusion, or during treatment with inotropes.

\section{Vasopressin antagonists}

Two vasopressin receptor antagonists, conivaptan $\left(\mathrm{V}_{1 \mathrm{a}}\right.$ and $\left.\mathrm{V}_{2}\right)$ and tolvaptan $\left(\mathrm{V}_{2}\right)$ are in clinical use. They cause vasodilation $\left(\mathrm{V}_{1}\right)$ and inhibit water-reabsorption $\left(\mathrm{V}_{2}\right)$. These drugs are indicated in the management of hyponatraemic hypervolaemia (present in over $20 \%$ of patients with AHF), but are not currently used in the UK for AHF.

\section{Adenosine-receptor antagonists}

Pre-existing renal failure and deteriorating renal function are common in patients with AHF. Adenosine itself is also thought to be an important mediator of renal dysfunction and diuretic resistance (adenosine reduces the glomerular filtration rate and stimulates renin release), so $\mathrm{A}_{1^{-}}$ receptor antagonists (e.g. rolofylline) have been investigated as therapies for improving dyspnoea, minimising renal deterioration and reducing fluid overload. However, trials have been disappointing.

\section{Digoxin}

Heart conditions have been treated with foxglove derivatives since 1785. Digoxin, a cardiac glycoside, has a relatively rapid onset and improves cardiac haemodynamics whilst avoiding many of the negative effects of hypotension, neurohormonal activation and renal failure. Digoxin may be useful in patients with HF who are in sinus rhythm ${ }^{3}$, but few definitive data are available.

\section{Summary and future advances}

Clinical trials investigating the treatment of AHF have been disappointing. RCTs of most drugs have only demonstrated improvements in haemodynamics but not long-term outcomes or survival. Furthermore, the traditional therapies of diuretics and dobutamine continue to be used despite little supportive trial evidence. Newer drugs under investigation include istaroxime, relaxin and omecamtiv.

\section{Istaroxime}

Newer drugs under investigation include istaroxime, which inhibits $\mathrm{Na}^{+} / \mathrm{K}^{+}$-ATPase and increases activity of $\mathrm{Ca}^{2+}$-ATPase at the sarcoplasmic reticulum, resulting in positive inotropy and lusitropy (myocardial relaxation) without increasing myocardial oxygen demand. Initial trials have demonstrated improvements in haemodynamics, and decreased diastolic stiffness in patients with acute heart failure but further data are needed.

\section{Relaxin}

Relaxin is a pregnancy hormone that antagonizes endothelin-1 by increasing the expression of endothelin-type $B$ receptors to cause selective vasodilation in blood vessels that are vasoconstricted. A recent study showed reduced symptoms and 180-day mortality in patients with AHF. ${ }^{4}$

\section{Omecamtiv mecarbil}

Omecamtiv mecarbil is a cardiac myosin activator that increases systolic ejection time without decreasing left ventricular contractility. As a result, it increases stroke volume and cardiac output without increasing oxygen demand. In patients with stable CHF, omecamtiv was associated with 
dose and concentration-dependent improvements in cardiac function ${ }^{5}$ and randomized controlled trials in patients with AHF are planned.

\section{Drugs used in chronic heart failure}

Chronic heart failure (CHF) is a progressive disease, often interrupted by episodes of acute decompensation. Treatment is aimed at minimising these episodes and slowing the decline of myocardial function, as well treating and preventing complications associated with the failing heart. Mortality from $\mathrm{CHF}$ is high, with an estimated $50 \%$ of patients not surviving beyond four years.

Most therapies target systolic heart failure (LVEF $\leq 40 \%$ ), but one in three symptomatic patients will have primarily diastolic dysfunction, often termed heart failure with preserved ejection fraction (HFPEF). Lifestyle changes (exercise, reduced salt intake, nutrition, weight loss, smoking cessation, diabetic control) and symptom management form the mainstay of treatment for both. Drugs do have a key role in reducing mortality from systolic heart failure; HFPEF treatment is less effective, although the overall prognosis with diastolic dysfunction is better.

\section{Angiotensin-converting enzyme inhibitors (ACEIS)}

All patients with symptomatic CHF or with a left ventricular ejection fraction (LVEF) $\leq 40 \%$ should be treated with ACEIs (e.g. enalapril, ramipril). Inhibiting ACE prevents the synthesis of angiotensin II, inhibits aldosterone secretion and cardiac remodelling. Successive large-scale RCTs have demonstrated improvements in ventricular function, reduced hospital admissions, and increased survival, together with an improvement in symptoms and quality of life. Contra-indications to ACEI therapy are a history of angioedema, bilateral renal artery stenosis, severe aortic stenosis, hyperkalemia $\left(\mathrm{K}^{+}>5.0 \mathrm{mmol} / \mathrm{L}\right)$ and a serum creatinine greater than $220 \mu \mathrm{mol} / \mathrm{L}$. ACEls also causes cough in approximately $20 \%$ of patients as a result of reduced ACE-dependent metabolism of bradykinins.

\section{Beta-blockers}

Beta blockade reduces death from cardiovascular disease. Despite negative chronotropic and inotropic effects, selective $\beta 1$-receptor blockers (e.g. bisoprolol, metoprolol) and carvedilol $\left(\beta_{1}, \beta_{2}\right.$ and $\alpha_{1}$-blocker) improve left ventricular function, symptoms and survival. Beta-blockers are antiarrhythmic, lower heart rate, improve diastolic function and reduce myocardial oxygen demand. They also reduce plasma concentrations of circulating catecholamines, renin and endothelin.

Beta blockers are indicated for patients with symptomatic systolic HF, or asymptomatic systolic HF following myocardial infarction (MI). Caution is required in AHF, though evidence suggests that betablockers can be commenced safely. Continuing beta-blocker during acute episodes of decompensation is not harmful, and maintains adherence following hospital discharge.

\section{Angiotensin receptor blockers (ARBs)}

ARBs (e.g. losartan) are indicated for symptomatic systolic heart failure that has not improved with an ACEI and beta blocker. They are also indicated when ACEls are not tolerated. ARBs selectively block the binding of angiotensin II to the $\mathrm{AT}_{1}$ receptor, reducing vasoconstriction, sodium retention and noradrenaline release.

\section{Aldosterone antagonists}

Low dose aldosterone antagonists (e.g. spironolactone, eplerenone) should be considered in patients with severe heart failure (LVEF $\leq 35 \%$ with severe symptoms). Addition of these drugs reduces hospital admissions and increase survival. There is probably some benefit even with mild 
symptoms and one recent study found greater eplerenone had greater efficacy compared with ARBs. ${ }^{6}$ Aldosterone antagonists reduce plasma volume and sodium retention. They also increase noradrenaline uptake, reduce myocardial oxidative stress and coronary artery inflammation, and reduce heart rate variability and ventricular remodelling. Aldosterone antagonists can worsen renal function and induce hyperkalaemia. Serial monitoring of electrolytes and renal function is mandatory, and as with ACEls, aldosterone antagonists should be avoided where there is hyperkalaemia or abnormal renal function.

\section{Diuretics}

Diuretics provide symptomatic relief from pulmonary and systemic venous congestion, but do not improve long-term survival (except where there are anti-aldosterone effects). Diuretics activate the renin-angiotensin system so should be used in combination with ACEls or ARBs. Loop diuretics are preferable as they are more effective at producing a diuresis and natriuresis. Electrolytes and renal function should be monitored before and during treatment.

\section{Hydralazine and isosorbide dinitrate}

Hydralazine and isosorbide dinitrate (H-IDN), a combination of an arterial and venodilator, has a limited role in the treatment of CHF. H-IDN may be beneficial in patients with persistent symptoms of heart failure despite maximal conventional therapy or when there is intolerance to ACEls or ARBs.

\section{Digoxin}

Digoxin is mostly used in the management of tachyarrhythmias, particularly atrial fibrillation. There is some evidence that, in symptomatic patients with LVEF of $\leq 40 \%$, symptoms, quality of life and number of hospital admissions are improved with digoxin.

\section{Ivabradine}

Ivabradine is a novel selective cardiac pacemaker current inhibitor that reduces heart rate. It has has been associated with improved outcome in patients with heart failure if used either alone or in combination with dobutamine, when it limits tachycardia.

\section{Summary}

The main treatments for the management of CHF are ACEIs and beta-blockers; the other therapies described above may be used as second line therapies. Newer pharmacological targets (endothelin receptor antagonists, prostacyclin analogues) have not been shown to improve survival in CHF.

There is no proven treatment for HFPEF. Minimising atrial tachyarrhythmias and minimising myocardial oxygen demand are key. Caution is required with the ACEls, nitrates or diuretics as too great a reduction in preload may result in decompensation. Future therapies include stem cell transplantation or targeting hormone and cytokine cell signalling.

\section{Coronary Insufficiency}

Coronary insufficiency occurs when myocardial oxygen demand exceeds its supply. It is caused by increases in demand (e.g. tachyarrhythmias) or reductions in supply (e.g. coronary artery disease) leading to symptoms of angina..Where reduced supply is due to artherosclerotic plaque formation, plaque rupture and subsequent thrombus formation leads to complete or partial artery occlusion resulting in acute myocardial infarction (MI). The management of acute $\mathrm{MI}$ is outside the scope of this article.

Coronary artery disease is the commonest presentation of coronary insufficiency, responsible for 80,568 UK deaths in 2010 . The aim of therapy is to maintain coronary artery perfusion, either by 
reducing myocardial oxygen demand or by maintaining or improving oxygen supply. Nitrates and beta-blockers are key to management. Beta-blockers are effective at treating symptoms (i.e. angina) and improving mortality in cardiovascular disease; nitrates provide only symptomatic relief.

\section{Anti-thrombotic agents}

These drugs stabilise atherosclerotic plaques, preventing thrombus formation. Inhibiting platelet activation impairs aggregation and thrombosis. Aspirin irreversibly inhibits the platelet enzyme cyclo-oxygenase 1 and hence prevents the production of thromboxane, a key component of platelet aggregation. At doses between $75-150 \mathrm{mg}$ per day, aspirin is well proven to prevent death from cardiovascular disease with an acceptably low risk of upper gastrointestinal bleeding.

The thienopyridines (e.g. clopidogrel, ticlodipine, prasugrel) are non-competitive ADP-receptor $\left(\mathrm{P}^{2} \mathrm{Y}_{12}\right)$ antagonists that block activation of the glycoprotein Ilb/Illa complex, so inhibiting platelet aggregation. They are used in combination with aspirin primarily to prevent stent-occlusion following coronary artery stent insertion. The metabolism of clopidogrel to its active metabolite is highly variable - clopidogrel may have a minimal therapeutic effect in poor metabolisers. Prasugrel is less affected by differences in metabolism, and is better than clopidogrel at preventing stent thrombosis and reducing non-fatal MI occurrence. However, the risk of major (including lifethreatening) bleeding is significantly increased.

Ticagrelor is a new class of drug that is a direct-acting, reversible $\mathrm{P}_{2} \mathrm{Y}_{12}$ inhibitor. It has more rapid and potent inhibitory effects on platelets compared to high-loading-dose clopidogrel, and also has a very quick offset, with rapid recovery of platelet function. Studies suggest that it ticagrelor is more effective than clopidogrel at reducing cardiovascular death after ACS and preventing stent thrombosis. Unlike prasugrel, major haemorrhage events do not appear to be significantly increased.

Other anti-thrombotic drugs that inhibit glycoprotein IIb/IIla include specific antibodies (e.g. abciximab), cyclic peptides (e.g eptifibatide) and non-peptide antagonists (e.g. tirofiban). These are all used in the management of acute coronary syndromes and in preventing thrombus formation after cardiac stent insertion.

\section{Statins}

Statins (e.g. simvastatin, atorvastatin) are HMG-Co-A reductase inhibitors, used for primary and secondary prevention of MI. Statins lower serum lipids, but also have anti-inflammatory properties related and unrelated to their lipid-lowering ability. Statins are associated with impaired endothelialleukocyte adhesion, inhibition of T-cell activation, impaired chemokine expression, as well as impaired synthesis of cell-signalling components. Modulation of the pro-inflammatory transcription factor NF-KB has also been implicated. Platelet aggregation is also impaired via such mechanisms as reduced thromboxane and increased prostacyclin expression. 


\section{References}

1. McMurray JJ, Adamopoulos S, Anker SD, et al. ESC Guidelines for the diagnosis and treatment of acute and chronic heart failure 2012: The Task Force for the Diagnosis and Treatment of Acute and Chronic Heart Failure 2012 of the European Society of Cardiology. Developed in collaboration with the Heart Failure Association (HFA) of the ESC. Eur Heart J 2012; 33: 1787-847.

2. O'Connor CM, Starling RC, Hernandez AF, et al. Effect of nesiritide in patients with acute decompensated heart failure. New Eng J Med 2011; 365: 32-43.

3. Hood WB, Jr., Dans AL, Guyatt GH, Jaeschke R, McMurray JJ. Digitalis for treatment of heart failure in patients in sinus rhythm.Cochrane Database Syst Rev 2014 Apr 28;4: CD002901

4. Teerlink JR, Cotter G, Davison BA, et al. Serelaxin, recombinant human relaxin-2, for treatment of acute heart failure (RELAX-AHF): a randomised, placebo-controlled trial. Lancet 2013; 381: 29-39.

5. Cleland JG, Teerlink JR, Senior R, et al. The effects of the cardiac myosin activator, omecamtiv mecarbil, on cardiac function in systolic heart failure: a double-blind, placebo-controlled, crossover, dose-ranging phase 2 trial. Lancet 2011; 378: 676-83.

6. Zannad F, McMurray JJ, Drexler H, et al. Rationale and design of the Eplerenone in Mild Patients Hospitalization And Survlval Study in Heart Failure (EMPHASIS-HF). Eur J Heart Failure 2010; 12: 61722.

\section{Further Reading}

1. Hamm CW, Bassand JP, Agewall S, et al. ESC Guidelines for the management of acute coronary syndromes in patients presenting without persistent ST-segment elevation: The Task Force for the management of acute coronary syndromes (ACS) in patients presenting without persistent ST-segment elevation of the European Society of Cardiology (ESC). Eur Heart J 2011; 32: 2999-3054.

2. McKelvie RS1, Moe GW, Ezekowitz JA et al. The 2012 Canadian Cardiovascular Society heart failure management guidelines update: focus on acute and chronic heart failure. Can J Cardiol. 2013; 29: 168-81.

3. Ramani GV, Uber PA, Mehra MR. Chronic Heart Failure: Contemporary Diagnosis and Management. Mayo Clin Proc 2010; 85: 180-95.

4. Khan SS, Gheorghiade M, Dunn JD, Pezalla E, Fonarow GC. Managed care interventions for improving outcomes in acute heart failure syndromes. Am J Manag Care 2008; 14: S273-86.

5. Givertz MM, Teerlink JR, Albert NM ET AL. Acute decompensated heart failure: update on new and emerging evidence and directions for future research. J Card Fail. 2013; 19: 371-89. 
Legend to Figure 1: Reduced ejection fraction (EF) varies, depending on the degree of diastolic and systolic dysfunction (EDV, end diastolic volume; ESV, end systolic volume), both tend to co-exist. Heart failure activates compensatory neurohumoral mechanisms within the vasculature and kidneys. Left unchecked, these mechanisms worsen cardiac function, with increased fluid retention increasing venous return (preload), and peripheral vasoconstriction increasing afterload. Pharmacological targets are indicated. 